\title{
Following One's Heart: Cardiac Rhythms Gate Central Initiation of Sympathetic Reflexes
}

\author{
Marcus A. Gray, ${ }^{1,2}$ Karin Rylander, ${ }^{4}$ Neil A. Harrison, ${ }^{3}$ B. Gunnar Wallin, ${ }^{4}$ and Hugo D. Critchley ${ }^{1}$ \\ ${ }^{1}$ Clinical Imaging Sciences Centre, Brighton and Sussex Medical School, University of Sussex, Brighton, East Sussex BN1 9RR, United Kingdom, ${ }^{2}$ Wellcome \\ Trust Centre for Neuroimaging, University College London, London WC1N 3BG, United Kingdom, ${ }^{3}$ Institute of Cognitive Neuroscience, University College \\ London, London WC1N 3AR, United Kingdom, and ${ }^{4}$ Institute of Neuroscience and Physiology, Department of Clinical Neurophysiology, Sahlgren \\ University Hospital, SE-413 45 Gothenburg, Sweden
}

Central nervous processing of environmental stimuli requires integration of sensory information with ongoing autonomic control of cardiovascular function. Rhythmic feedback of cardiac and baroreceptor activity contributes dynamically to homeostatic autonomic control. We examined how the processing of brief somatosensory stimuli is altered across the cardiac cycle to evoke differential changes in bodily state. Using functional magnetic resonance imaging of brain and noninvasive beat-to-beat cardiovascular monitoring, we show that stimuli presented before and during early cardiac systole elicited differential changes in neural activity within amygdala, anterior insula and pons, and engendered different effects on blood pressure. Stimulation delivered during early systole inhibited blood pressure increases. Individual differences in heart rate variability predicted magnitude of differential cardiac timing responses within periaqueductal gray, amygdala and insula. Our findings highlight integration of somatosensory and phasic baroreceptor information at cortical, limbic and brainstem levels, with relevance to mechanisms underlying pain control, hypertension and anxiety.

Key words: baroreceptor; blood pressure; PAG; amygdala; insula; fMRI; shock; anxiety; blood phobia; HF HRV

\section{Introduction}

The processing of environmental stimuli is influenced by visceral state. The brain receives continuous afferent information about internal bodily functions. Cardiovascular homeostasis depends on such feedback; notably phasic signals from aortic arch and carotid sinus baroreceptors activated by blood-volumedistension during cardiac systole (Mancia and Mark, 1983). Baroreceptor firing modulates efferent autonomic responses in an organ-specific manner, inhibiting muscle sympathetic nerves while leaving skin sympathetic outflow unaltered (Wallin, 2007). The central processing of painful stimuli is influenced by experimentally increasing aortic baroreceptor firing via external neck pressure at specific phases of the cardiac cycle, reducing perceived pain and reflexive responses (e.g., limb withdrawal) (Brody and Rau, 1994; Droste et al., 1994; Dworkin et al., 1994; Kardos et al., 1994; Mini et al., 1995; Angrilli et al., 1997; Edwards et al., 2001, 2002; Rau and Elbert, 2001).

Sympathetic outflow to muscle vascular beds [muscle sympathetic nerve activity (MSNA)] occurs in characteristic bursts, reflecting the cyclical inhibition of efferent brainstem cardiovascular nuclei, including the rostral ventrolateral medulla (RVLM) by

Received July 18, 2008; revised Dec. 4, 2008; accepted Dec. 7, 2008.

This work was performed at the Wellcome Trust Centre for Neuroimaging (WTCN), Queen Square, London, and supported by a Wellcome Trust Programme Grant (H.D.C.), a Swedish Medical Research Council Grant (12170), and funding to the WTCN. We thank Peter Aston and Eric Featherstone at the WTCN for help with the recording of physiological data within the fMRI environment.

Correspondence should be addressed to Marcus A. Gray, Clinical Imaging Sciences Centre, Brighton and Sussex Medical School, University of Sussex, Brighton, East Sussex BN1 9RR, UK. E-mail: m.a.gray@bsms.ac.uk. DOI:10.1523/JNEUROSCI.3363-08.2009

Copyright $\odot 2009$ Society for Neuroscience $\quad 0270-6474 / 09 / 291817-09 \$ 15.00 / 0$ baroreceptor feedback (McAllen and Malpas, 1997; Jänig, 2006). Unexpected somatosensory stimuli occurring during early cardiac systole [200-400 ms after electrocardiogram (ECG) R-wave] further inhibit MSNA in $\sim 60 \%$ of healthy subjects (Donadio et al., 2002a,b; Eder et al., 2009). In contrast, stimuli presented at the ECG R-wave (hereafter called synchronous stimuli), i.e., before the systolic pressure wave, do not inhibit MSNA (Donadio et al., 2002a,b; Wallin, 2007). Thus, when stimuli are administered after a delay relative to the ECG R-wave (hereafter delayed stimuli), baroreceptor impulses inhibit activity in sympathetic nerves supplying muscle. This inhibition is exaggerated in patients with blood phobia and syncope, suggesting that feedback mechanisms supporting cardiac homeostasis shape an individual's affective style (Donadio et al., 2007). Stimulus timing within the cardiac cycle also influences reflexive and/or subjective impact of somatosensory stimulation; limb withdrawal and painful sensation are reduced to delayed versus synchronous stimuli (Elbert et al., 1988; Dworkin et al., 1994; Angrilli et al., 1997; Edwards et al., 2002, 2003, 2008).

The role of baroreceptor-sensitive brainstem nuclei in the homeostatic regulation of blood pressure is well characterized (Gilbey and Spyer, 1993; McAllen and Malpas, 1997; Spyer, 1999; Jänig, 2006; Gilbey, 2007). However, the mechanisms though which baroreceptor activity influences sensory and emotional processing is poorly understood. Neuroimaging studies implicate anterior insular and anterior cingulate cortex (ACC) in mediating cardiac influences on conscious feeling states (Craig, 2002; Critchley, 2005; Gianaros et al., 2007). The amygdala, via direct connections with pontine autonomic nuclei, is further implicated in translating psychological stress into bodily arousal, including 
patterns of blood pressure reactivity (Gianaros et al., 2008). We therefore hypothesized the activity of brainstem autonomic nuclei, amygdala, anterior insula and ACC would reflect cardiac timing influences on physiological reactions to somatosensory stimuli. We simultaneously recorded neural activity and physiological (beat-to-beat blood pressure) responses to unpredictable electrical stimulation of the skin at different times within the cardiac cycle to investigate the central mechanisms through which sensory and baroreceptor information interact.

\section{Materials and Methods}

Participants. Eleven healthy right-handed adults ( 7 female, 4 male, mean \pm SD age $27.2 \pm$ 7.2 years) with no history of psychiatric, neurological or cardiovascular illness or psychotropic or cardiovascular medication gave informed written consent to take part in this study which was approved by the Joint Research Ethics Committee of the Institute of Neurology and the National Hospital for Neurology and Neurosurgery, Queen Square, UK. Informed consent was obtained after full explanation of the study and procedures.

Procedure. The scanning study was designed to investigate ECG R-wave synchronous and delayed stimuli. Immediately before scanning, ECG and pulse oximetry (Nonin 8600 FO Series, Nonin Medical Inc.) data were recorded in a psychophysiology laboratory with the participant in a reclined position, to determine mean pulse transit time, calculated as time between ECG R-wave and peak finger pulse oximetry waveform. In the MRI scanner, participants were then fitted with three ECG electrodes (mid sternum, $5 \mathrm{~cm}$ above and below left nipple). An inflatable finger cuff and infrared plethysmograph were fitted to the index finger of the subject's left hand, allowing measurements of beatto-beat finger arterial blood pressure with a Portapres device (Finapres Medical Systems) adapted in-house to be MR compatible. Finally, the electrocutaneous shock electrode was applied to the back of the right hand using a customised optically isolated MR compatible system incorporating a Digitimer DS7A stimulator. The stimulation electrode consisted of a central cathode (diameter $0.5 \mathrm{~mm}$ ) surrounded by a ring anode (diameter $6 \mathrm{~mm}$ ) (Katsarava et al., 2006), providing a stimulation area of $19.6 \mathrm{~mm}^{2}$. Participants underwent functional magnetic resonance imaging (fMRI) of the brain while synchronous or delayed electrical shocks were delivered to the dorsum of the hand.

ECG, skin conductance response (SCR), and Portapres (beat-to-beat blood pressure) analog waveform data were transformed into frequency data for fiber optic transmission from the MRI scanning room (1000, 10,000 , and $50,000 \mathrm{~Hz}$ respective frequencies) to minimize radio frequency interference. Because scanner artifacts contaminated the ECG signal and could not be filtered away in real time, we used the pulse oximetry signal to trigger stimulus delivery relative to the next predicted ECG R-wave. The relationship between the times of stimulus presentation and ECG R-waves was validated post hoc.

Task and design. Participants were informed of the study aims; to examine neural and physiological responses to electrical skin stimuli. Seventy-two unpredictable (pseudorandomized presentation) electric shocks (mean ISI $26.1692 \pm 23.289$ s) were timed to occur either syn- chronous with the ECG R-wave (estimated using mean pulse transit time from preceding pulse oximetry waveform) or delayed [100-500 ms (mean 284.8, SD = 105.1 ms) after the ECG R-wave (see Fig. 1). Shocks were $10 \mathrm{~ms}$ square waves, set at a subject defined intensity of "strong, but not painful" (mean intensity $5 \mathrm{~mA}, 60 \mathrm{~V}$ ). Participants were instructed to simply focus on a central fixation cross and wait for sensory stimuli. On average, each subject was presented with 27.1 synchronous $(S D=6.59$ ) and 29.5 delayed shocks $(S D=8.13)$.

MRI acquisition and image preprocessing. Functional echo-planar datasets sensitive to BOLD (blood oxygen level dependent) contrast were acquired at 3 Tesla (Siemens Alegra) continuously throughout the stimulus delivery. The functional sequence minimized orbitofrontal signal dropout (Deichmann et al., 2003) and achieved partial brain coverage (40 slices, $1.5 \mathrm{~mm}$ slice thickness no gap, tilted $30^{\circ}$ from intercommissural plane (see Fig. 3A) (TR $102 \mathrm{~ms}$, TE $30 \mathrm{~ms}$ ). In addition, a full brain T2 weighted volume (identical parameters, 100 slices), a full brain B0 phase-map (64 slices $128 \times 128$ matrix) and a high resolution T1weighted structural scan $\left(1 \mathrm{~mm}^{3}\right)$ were acquired to optimize functional unwarping and normalization. Extensive physiological monitoring (beat-to-beat blood pressure, GSR, ECG, pulse oximetry) and shock delivery resulted in a small increase in radio frequency (RF) noise. Occasional transitory artifacts appeared as clearly visible intensity increases within a small number of neighboring voxels, restricted to a single column in a single slice of an fMRI volume Functional images were therefore 
Mean Arterial Blood Pressure Responses

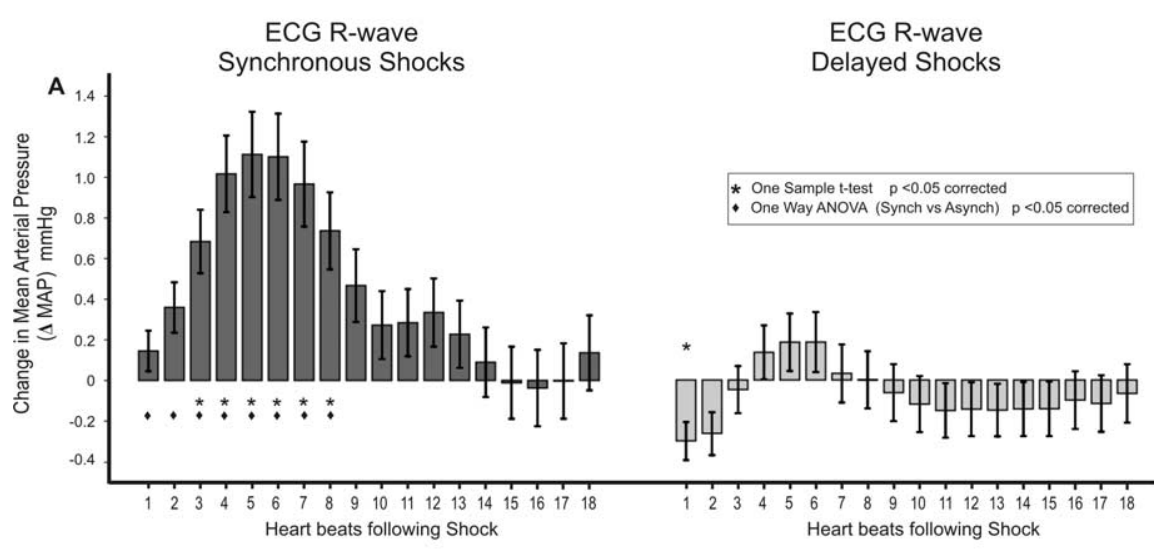

B

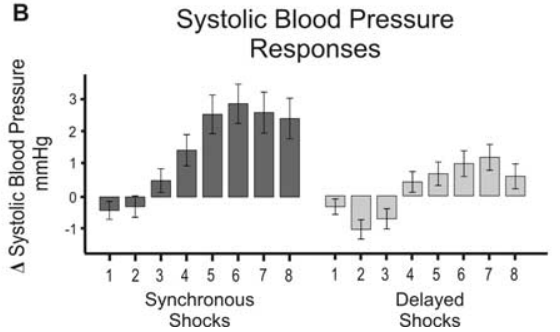

C

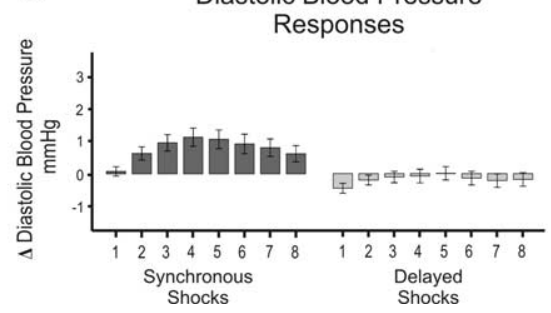

Figure 2. Blood pressure changes after electric shocks. $A$, MAP increases after synchronous shocks were significantly greater than those observed after delayed shocks. $\boldsymbol{B}, \boldsymbol{C}$, Similar effects were observed in systolic $(\boldsymbol{B})$ and diastolic $(\boldsymbol{C})$ blood pressure measures, with significantly greater increases observed after R-wave synchronous shocks. description, this numbering convention has been modified from previously used numberings focused on microneurography recordings (Donadio et al., 2002b, 2007). All blood pressure data are presented as change from baseline (mean of the respective blood pressure parameters between R-waves -4 to -1 ; Fig. 1, highlighted in red).

Heart rate variability analysis. Heart rate measures [interbeat interval (IBI)] were derived from pulse oximetry recordings covering the entire recording period ( $\sim 45 \mathrm{~min})$. Heart rate variability (HRV) was assessed by calculating the power spectral density (PSD) of both the entire IBI time series, and of $60 \mathrm{~s}$ epochs after each shock providing measures of heart rate change within very low $(0-0.04)$, low $(0.04-$ $0.15)$ and high frequency bands (0.15-0.4). High frequency heart rate variability (HF HRV) provides an index of relative parasympathetic (primarily vagus nerve) influences on the heart (Berntson et al., 1993; Task Force, 1996). Spectral density estimates were calculated using autoregressive time series modeling in HRV analysis tool version 1.1 (http://bsamig.uku.fi/ index.shtml, Department of Physics, University of Kuopio, Finland). To explore the physiological basis to individual differences in cardiac cycle effects of stimulation, we examined both total HF power during the entire recording, and change in HF power after delayed (relative to synchronous) shocks, deriving a single measure of each for each subject.

$f M R I$ data analysis. Region-of-interest (ROI) screened for RF spike artifacts, initially slice by slice and then column by column (phase encode direction) within each slice using a customwritten SPM5 toolbox. Columns containing spikes were replaced with the mean of surrounding columns. Second, we ran a voxel by voxel screen over each of the $\sim 20,000$ voxels in each brain volume, for all volumes per subject, for all subjects, with a custom written 2-dimensional median intensity filter. We set the threshold at $20 \%$ above or below extreme values in surrounding voxels, replacing outliers with the median (variable size rectangular kernel $=<9 \times 9$ voxels). Because the change in BOLD signal intensity due to brain activity is typically $2-3 \%$ of the total signal this was unlikely to alter uncontaminated data. These procedures were successful and conservative ( $<2 \%$ data replacement in total). Images were preprocessed using SPM5 (http://www.fil.ion.ucl.ac.uk/spm), using spatial realignment and unwarping incorporating B0 field mapping (Hutton et al., 2002) and sequential coregistration (6 parameter rigid body spatial transformation). Structural scans were segmented into CSF, gray and white matter images and iteratively normalized to standard space (Montreal Neurologic Institute) using a single generative model (Ashburner and Friston, 2005). Transformation parameters for structural image segmentation and normalization were then applied to transforming participants' functional data into normalized images in standard space. Functional scans were subsequently smoothed with an 8 $\mathrm{mm}$ Gaussian smoothing kernel. The first six functional volumes were discarded to allow for equilibration of net magnetization

Physiological data analysis. Beat-to-beat pressure waveforms obtained from the finger arterial pressure waveform recorded on the Portapres and reconstructed as brachial pressures. A set of physiological measures, including brachial pressure at systole and diastole, and mean arterial pressure (MAP) (calculated as the integral of the brachial pressure curve/ number of measurements during that ECG cycle) was derived from these smoothed waveforms.

Stimuli were triggered by the ECG R-wave labeled the stimulus R-wave (Fig. 1). Blood pressure waves generated between the stimulus beat and $\mathrm{R}$-wave 1 are not considered, because this period is confounded by shock delivery. The blood pressure wave generated during the following cycle cardiac cycle (from R-wave 1 to R-wave 2) is labeled BP 1 . For ease of masks were constructed for bilateral amygdala, bilateral anterior insula, ACC and brainstem. Insula ROIs consisted of two $22 \times 24 \times 28 \mathrm{~mm}$ rectangular masks (left centered at $-43,18,-4$, right centered at 48,18 , $-4)$ whereas the ACC ROI was a $20 \times 30 \times 30 \mathrm{~mm}$ rectangular mask centered at $0,16,36$. Brainstem ROI was a $32 \times 34 \times 30 \mathrm{~mm}$ rectangular mask (center $0,-25,-27$ ). Finally, the amygdala ROI was traced to include basolateral, central, and superficial nuclei. Family-wise error (FWE) corrections for multiple comparisons were made for each anatomical ROI based on random field theory and implemented in SPM5 (Friston et al., 1994).

\section{Results}

\section{Physiological timing effects}

We first examined how beat-to-beat blood pressure responses after somatosensory stimulation were affected by cardiac timing. MAP was significantly greater during the first 8 cardiac cycles after synchronous shocks when compared with delayed shocks (Fig. 2A, Table 1) (supplemental Table S1, available at www. jneurosci.org as supplemental material). Similarly, larger increases in systolic and diastolic blood pressure were observed after synchronous compared with delayed shocks (Fig. $2 B, C$ ) (supplemental Tables S2 and S3, available at www.jneurosci.org as supplemental material). Heart rate was not significantly altered by the occurrence or timing of stimuli [IBI (group mean \pm $\mathrm{SD})=874.16 \pm 149.38 \mathrm{~ms}]$.

We also examined the effects on blood pressure within subjects, motivated by the finding that there is variability across subjects in the degree to which delayed shocks inhibit sympathetic nerve outflow to the muscle (Wallin, 2007). Significant differences were observed at an individual level in three participants and trends in the same direction were observed in four others (see supplemental Fig. S1, available at www.jneurosci.org as supplemental material). 
Table 1. Differences between blood pressure (MAP) responses after synchronous versus delayed shocks

\begin{tabular}{|c|c|c|c|c|c|c|}
\hline Dependent variable & $d f$ & Mean square & Levene's statistic & Levene's significance & $F$ & $\begin{array}{l}\text { Significance } \\
(\alpha=0.0028)\end{array}$ \\
\hline MAP 1 & 1,690 & 33.243 & 0.904 & 0.342 & 10.500 & $0.001^{*}$ \\
\hline MAP 2 & 1,694 & 65.575 & 0.284 & 0.594 & 14.794 & $0.000 *$ \\
\hline MAP 3 & 1,695 & 90.410 & 0.271 & 0.603 & 14.828 & $0.000^{*}$ \\
\hline MAP 4 & 1,700 & 132.208 & 1.501 & 0.221 & 15.485 & $0.000^{*}$ \\
\hline MAP 5 & 1,700 & 146.320 & 3.265 & 0.071 & 14.360 & $0.000 *$ \\
\hline MAP 6 & 1,698 & 142.232 & 1.773 & 0.183 & 13.353 & $0.000^{*}$ \\
\hline MAP 7 & 1,694 & 147.760 & 3.706 & 0.055 & 14.533 & $0.000^{*}$ \\
\hline MAP 8 & 1,692 & 91.250 & 0.792 & 0.374 & 10.121 & $0.002 *$ \\
\hline MAP 9 & 1,692 & 47.345 & 0.486 & 0.486 & 5.573 & 0.019 \\
\hline MAP 10 & 1,689 & 25.605 & 0.041 & 0.840 & 3.273 & 0.071 \\
\hline MAP 11 & 1,691 & 31.699 & 0.004 & 0.948 & 4.217 & 0.040 \\
\hline MAP 12 & 1,688 & 38.119 & 0.062 & 0.803 & 5.081 & 0.025 \\
\hline MAP 13 & 1,688 & 23.583 & 0.214 & 0.643 & 3.267 & 0.071 \\
\hline MAP 14 & 1,682 & 8.956 & 0.462 & 0.497 & 1.176 & 0.278 \\
\hline MAP 15 & 1,680 & 2.810 & 1.654 & 0.199 & 0.353 & 0.553 \\
\hline MAP 16 & 1,669 & 0.615 & 2.340 & 0.127 & 0.070 & 0.791 \\
\hline MAP 17 & 1,667 & 2.073 & 4.294 & 0.039 & 0.247 & 0.619 \\
\hline MAP 18 & 1,646 & 6.444 & 2.586 & 0.108 & 0.771 & 0.380 \\
\hline
\end{tabular}

One-way ANOVA revealed synchronous shocks evoked significantly greater MAP increases than delayed shocks. Highlighted (boldface) results are significant after correction for multiple comparisons ( $\alpha=0.0028)$. Nonsignificant Levene's statistics demonstrate equality of variances.

Significantly increased MAP responses following synchronous shocks remained after excluding clear nonresponders.

\section{CNS responses}

Neuroimaging data, quantifying BOLD signal changes across the scanning session, were analyzed using multiple regression analyses to identify changes in regional neural responses during the processing of synchronous and delayed shock that evoked distinct effects on peripheral blood pressure. Our model included separate regressors of interest identifying neural activity mediating beat-to-beat MAP responses to synchronous and delayed shocks for seven seconds after each stimulus, convolved with a delayed hemodynamic response function to account for the temporal relationship between neural activity and BOLD signal. We stringently controlled for artifactual (i.e., independent of neural activity) influences of blood pressure on BOLD measurements by including immediate (nondelayed and nonconvolved) MAP changes as a continuous nuisance regressor within the same model.

Our results identify differential responses within anterior insula, amygdala and brainstem to synchronous and delayed shocks (Fig. 3, Table 2). The differential neural responses within these a priori regions of interest are FWE corrected for the bilateral volume of each structure. Evoked activity within left anterior insula and mid pons was significantly greater after synchronous rather than delayed stimuli. A similar response pattern within the right insula was observed at lower (uncorrected) significance. Conversely, within the right amygdala (in the region of the central nucleus), activity increased after delayed and decreased after synchronous stimuli (Fig. 3). Again, significant differential responses within anterior insula, amygdala and brainstem remained after excluding clear nonresponders.

We also examined whether individual differences in vagal tone predicted differences in blood pressure or neural responses after synchronous versus delayed stimuli. The relationship between individual differences in evoked blood pressure responses and individual differences in neural or HRV measures did not reach significance (see supplemental Fig. S2, available at www. jneurosci.org as supplemental material). However second level covariate analyses revealed strong associations between differen- tial neural responses and HRV. Interindividual differences in overall HF HRV power were significantly associated with interindividual differences in BOLD response within the left insula (Fig. 4D, E, Table 3, top); greater HF power was associated with a larger functional increase after synchronous than after delayed shocks (clusters closely match regions observed in Fig. 3B). Differential changes in HF power also reflected neural responses (Fig. 4F, G, Table 3, bottom). Increased HF power after delayed shocks was associated with increased right amygdala and periaqueductal gray activity (relative to synchronous shocks). Similar associations between HF power after delayed shocks were also observed within the anterior cingulate and left ventrolateral prefrontal cortices.

\section{Discussion}

The present study implicates specific cortical, limbic and brainstem centers for integration of responses to somatosensory with pulsatile baroreceptor stimulation. Specifically our main findings were: (1) delayed electric shocks delivered to the skin significantly attenuated MAP increases when compared with ECG R-wave synchronous shocks; (2) the central neural responses differed between synchronous and delayed shocks, with greater activity within insula and pons after synchronous and within right amygdala after delayed shocks; and (3) on an individual basis, differences in central neural responses between delayed and synchronous stimuli correlated closely to corresponding differences in HRV indices of vagal function.

Our findings regarding cardiovascular responses to electrical skin stimuli extend previous studies demonstrating that $\mathrm{R}$-wave delayed stimulation inhibits sympathetic vasoconstrictor outflow to the vascular bed of skeletal muscle but not to skin (Wallin, 2007), and that increased baroreceptor activation attenuates nociceptive somatomotor reflexes (Dworkin et al., 1994; Rau and Elbert, 2001; Edwards et al., 2002; McIntyre et al., 2006, 2008). Increases in blood pressure evoked by sensory stimuli arise mainly through sympathetically mediated vasoconstriction, the magnitude of which reflects the balance between excitatory and inhibitory effects on sympathetic vasoconstrictor nerves across the body. Recordings of peripheral blood flow and/or sympathetic nerve traffic show that unexpected sensory stimuli induce 


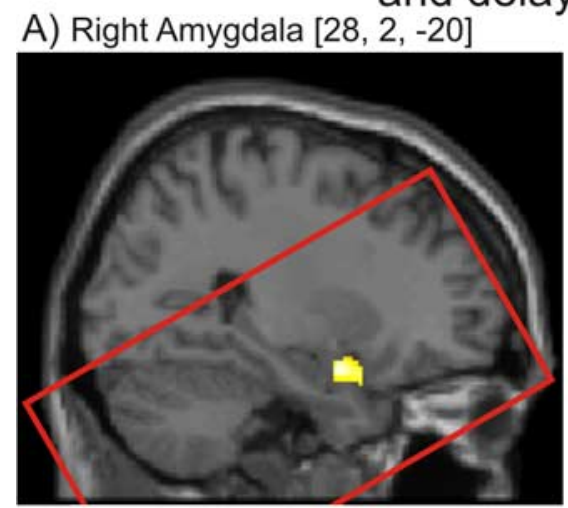

\section{Differential responses to synchronous and delayed shocks}

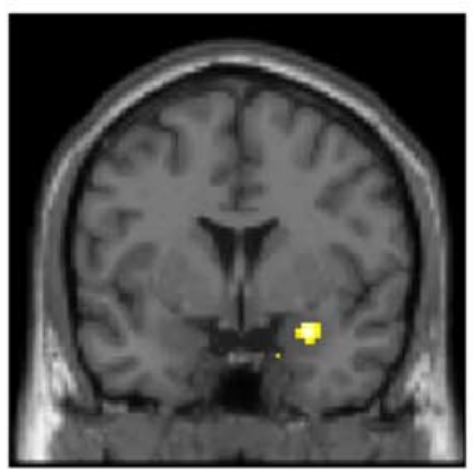

\section{B) Left Insula Cortex $[-34,12,-16]$}
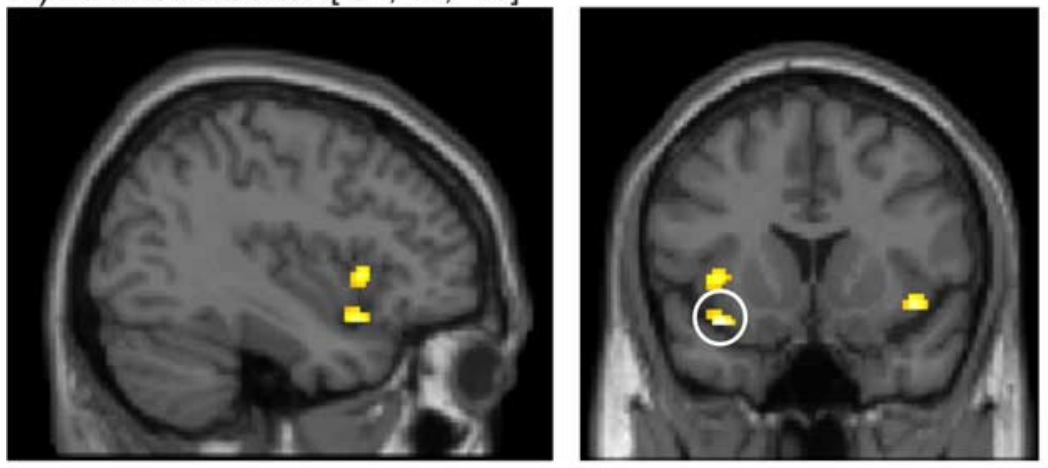

C) Brainstem $[-2,-14,-32]$
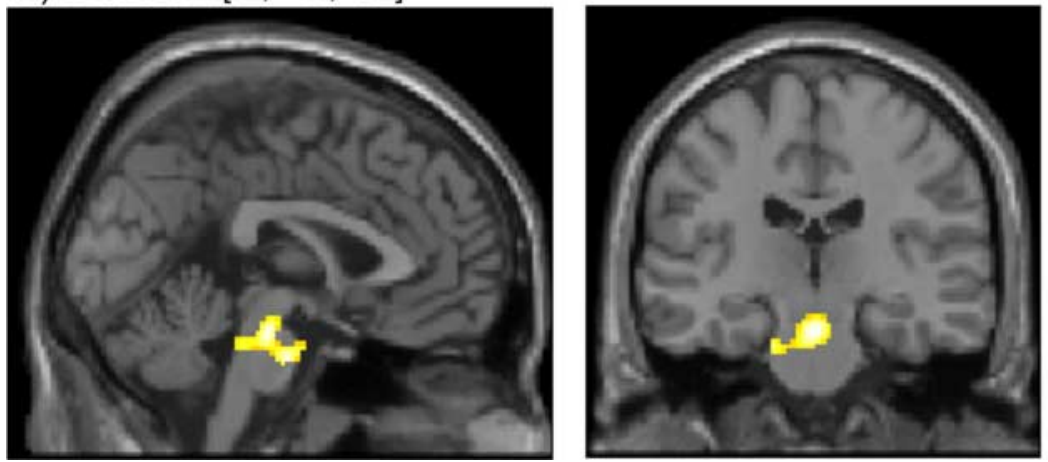

Figure 3. $\boldsymbol{A}-\boldsymbol{C}$, Differential responses to synchronous and delayed shocks. Right amygdala activity $(\boldsymbol{A})$ was significantly increased after delayed relative to synchronous shocks. Conversely, left anterior insula $(\boldsymbol{B})$ (cluster surviving ROI FWE correction circled) and brainstem autonomic nuclei $(\boldsymbol{C})$ activity was significantly greater after synchronous shocks. The red rectangle in $\boldsymbol{A}$ illustrates the extent of partial brain T2 data acquired.

Table 2. fMRI measures revealed differential responses depending on the timing of brief skin shocks in relation to ECG within right amygdala, left anterior insula, and brainstem nuclei

\begin{tabular}{|c|c|c|c|c|c|c|}
\hline Interaction of shock and ECG synchrony & $x$ & $y$ & $z$ & $t$ & $k(p<0.001)$ & ROI FWE $p$ \\
\hline Amygdala & 28 & 2 & -18 & 4.14 & 25 & 0.028 \\
\hline Anterior insula & -36 & 14 & -16 & 4.41 & 19 & 0.022 \\
\hline Brainstem & 0 & -22 & -22 & 4.14 & 43 & 0.033 \\
\hline Brainstem & -2 & -14 & -32 & 4.07 & 26 & 0.039 \\
\hline
\end{tabular}

transient vasoconstriction in the skin, kidney and splanchnic vascular beds (Forsyth, 1972; Caraffa-Braga et al., 1973; Yu and Blessing, 1997) but vasoconstriction is not typically observed in muscle, highlighting organ specific sympathetic control. In the
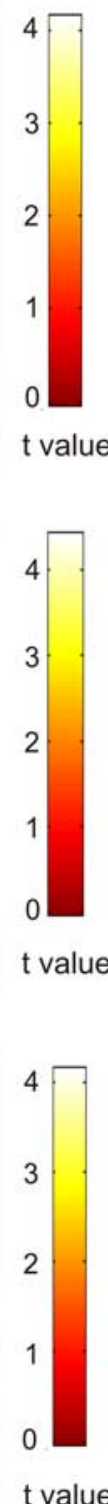

present study, the differences in MAP are likely to correspond to vasodilation within skeletal muscle consequent upon MSNA inhibition during delayed (but not synchronous) shocks, counteracting the vasoconstriction in other vascular beds (Jänig, 1985; Wallin, 2007).

A central finding is that cortical and limbic neural responses differed to shocks presented at different times in the cardiac cycle. Our data indicate the presence of a discrete matrix of brain regions including amygdala, insula and brainstem nuclei that support the integration of somatosensory information with phasic cardiovascular feedback to control autonomic arousal responses. Notably, the processing of delayed stimuli, coincident with the systolic peak of baroreceptor signaling, activated the amygdala and attenuated responses in bilateral insula and mid pons. Both insula and amygdala are sensitive to feedback from bodily states of arousal, yet in this experimental context the two regions showed distinct responses. A body of research relates insula activity to the representation and regulation of myocardial function (Oppenheimer, 2007). Previous functional imaging suggests differential insula response to cardiac timed stimuli during interoception (Critchley et al., 2004; Gray et al., 2007). We observed increased blood pressure after synchronous shock, without significantly altered heart rate, suggesting altered cardiac ventricular contractility and/or peripheral resistance. Autonomic innervation regulating cardiac dromotropy and inotropy (rapidity and force of ventricular contractility) is predominantly left sided (Wittling et al., 1998). Our finding of greater increases in left insula function after synchronous shocks may correspond to left sided cerebral regulation of cardiac contractility. However, the close association between the magnitude of this effect and overall HF power (generally accepted to index parasympathetic cardiac regulation) across subjects suggests that the functional differences may reflect the degree of parasympathetic inhibition after delayed shocks. An alternative explanation is that activity in paralimbic insula cortex reflects cortical re-representations of visceral and autonomic status (Craig, 2002; Critchley et al., 2004; Gray and Critchley, 2007) in contrast to the direct coupling of brainstem [periaqueductal gray (PAG)] (Kober et al., 2008) and amygdala with afferent baroreceptor inhibition of sympathetic cardiovascular reflexes.

An important earlier observation is that there are reproducible individual differences in the degree of MSNA inhibition to delayed shocks (Wallin, 2007). These differences may partially 

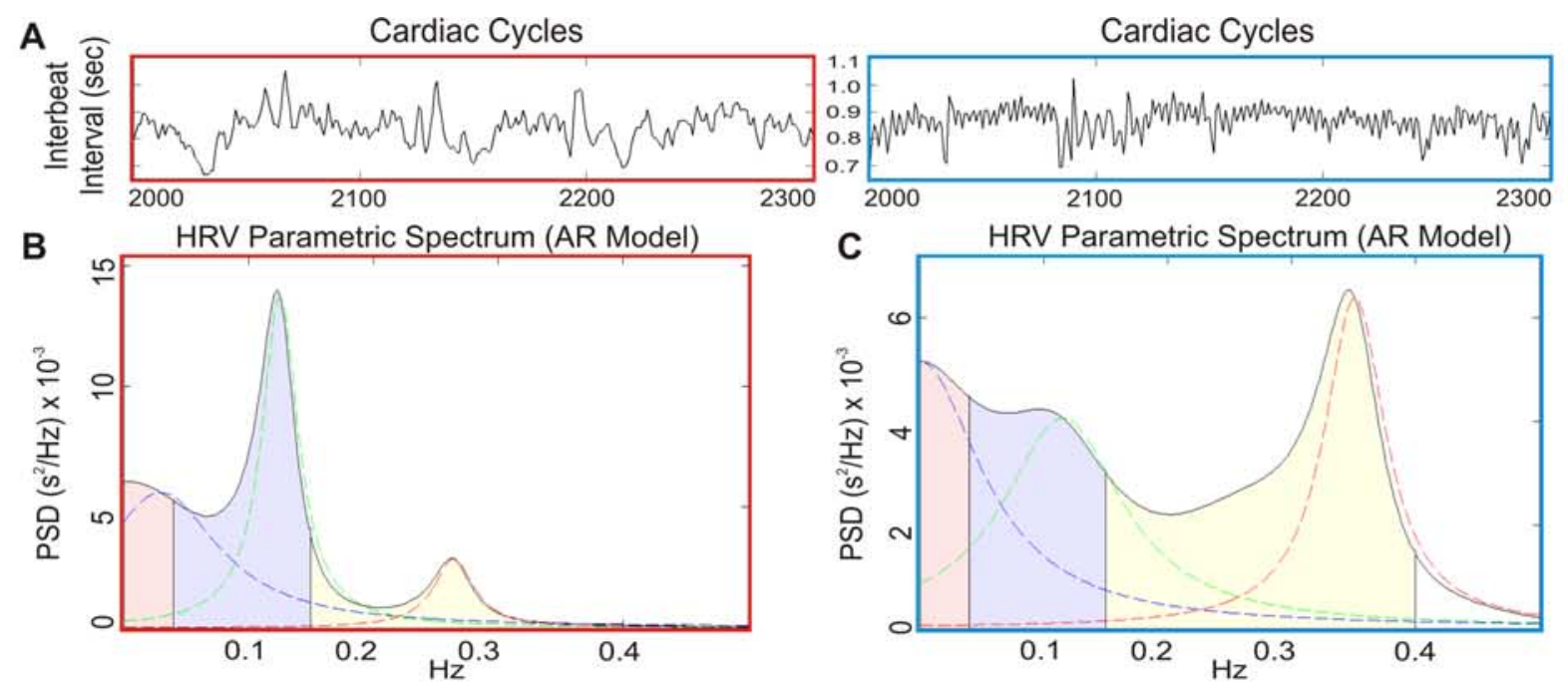

$\square$ Very Low Frequency $(0-0.04 \mathrm{~Hz}) \quad \square$ Low Frequency $(0.04-0.15 \mathrm{~Hz}) \quad \square$ HighFrequency $(0.15-0.4 \mathrm{~Hz})$

Total High Frequency Power Correlated with Differential Neural Response (Synchronous - Delayed )

D

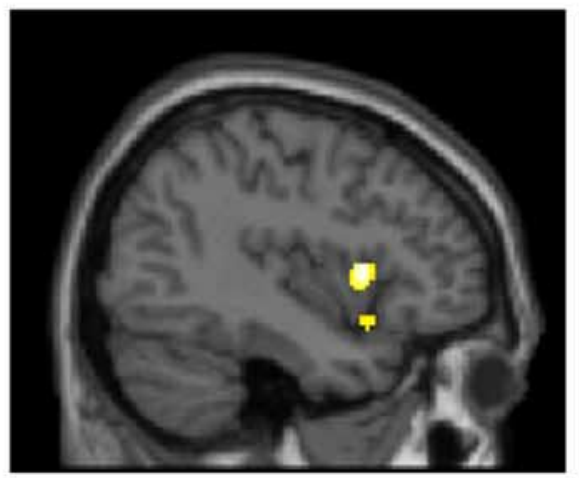

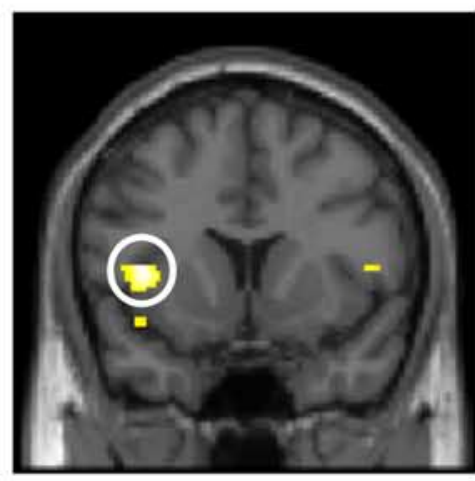

E

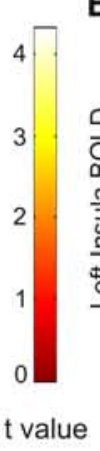

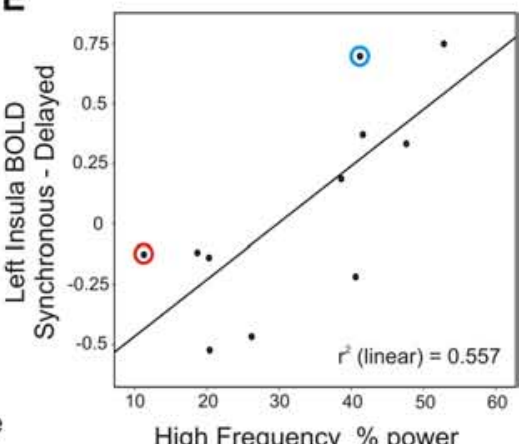

High Frequency Power Change Correlated with Neural Response Change (Delayed - Synchronous)

$\mathbf{F}$
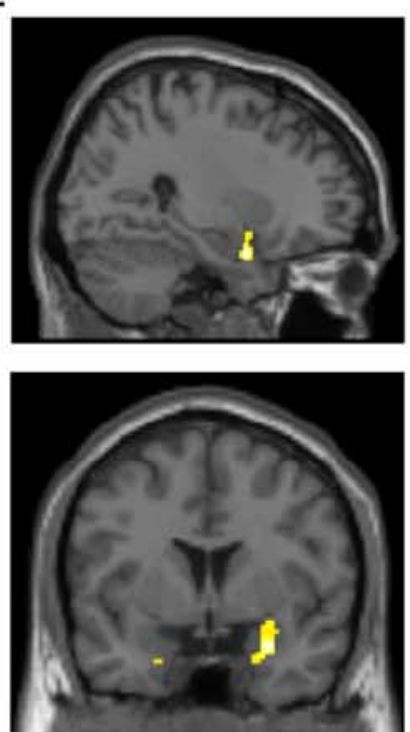

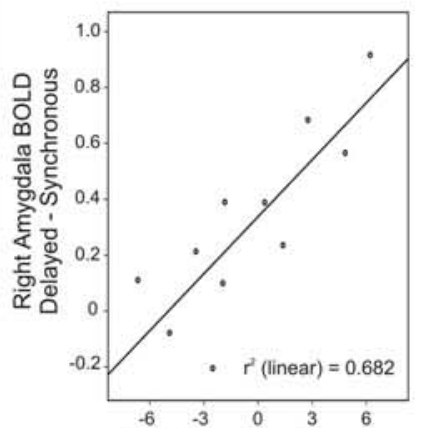

High Frequency \% powe Delayed - Synchronous

t value

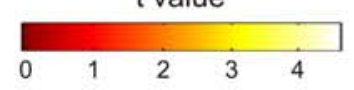

G
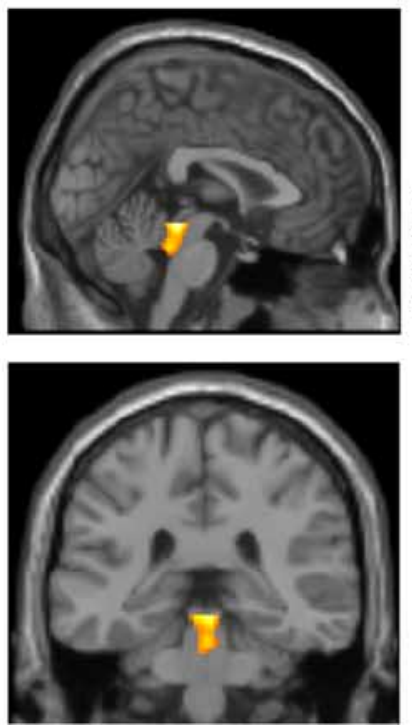

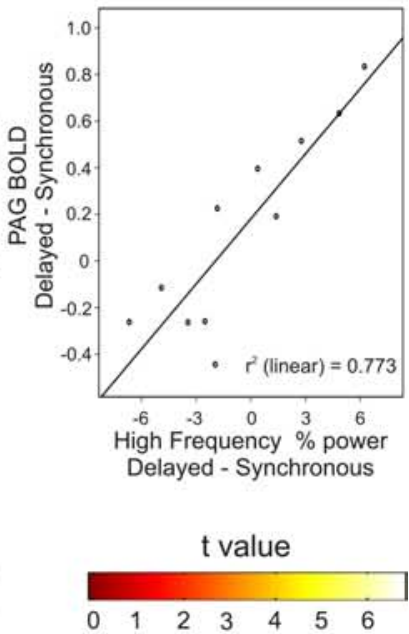

Figure 4. HRV predicts differential responses to synchronous and delayed shocks within left insula and brainstem. $\boldsymbol{A}$, The variability in interbeat intervals (only 300 beats illustrated) is displayed for two subjects. Parametric autoregressive PSD measures identify very-low-frequency, low-frequency, and high-frequency HRV components. $\boldsymbol{B}, \boldsymbol{C}, \mathrm{HRV}$ spectrums are shown for a subject with small ( $\boldsymbol{B}$, red bordered plots) and large ( $\boldsymbol{C}$, blue bordered plots) high-frequency components. $\boldsymbol{D}$, Across subjects, differential neural responses to shocks (synchronous- delayed) within the left insula correlate with HF HRV during the entire experiment. $\boldsymbol{E}$, Greater differences in insula response are observed with increased HF HRV (colored circles identify subjects displayed above). $\boldsymbol{F}, \boldsymbol{G}$, Changes in HF HRV correlate with changes in neural responses across subjects. Greater HF HRV after delayed shocks is significantly associated with greater BOLD response after delayed shocks in right amygdala $(\boldsymbol{F})$ and periaqueductal gray $(\boldsymbol{G})$. 
Table 3. HRV predicts differential responses to synchronous and delayed shocks

\begin{tabular}{|c|c|c|c|c|c|c|c|}
\hline & $x$ & $y$ & $z$ & $t$ & $k(p<0.001)$ & Correction & $p$ \\
\hline \multicolumn{8}{|c|}{ Total HF HRV and increased neural response to synchronous shocks } \\
\hline \multirow[t]{2}{*}{ Left insula } & -40 & 11 & 4 & 4.32 & 38 & FDR & 0.050 \\
\hline & & & & & & $\mathrm{FWE}_{\text {insula }}$ & 0.060 \\
\hline \multicolumn{8}{|c|}{ Increased HF HRV and neural response after delayed shocks } \\
\hline Right amygdala & 28 & 2 & -22 & 4.60 & 22 & $\mathrm{FWE}_{\mathrm{amy}}$ & 0.020 \\
\hline Periaqueductal gray & 2 & -32 & -12 & 6.80 & 91 & FWE & 0.005 \\
\hline Anterior cingulate cortex & -20 & 40 & -10 & 5.97 & 34 & FWE & 0.0330 \\
\hline Left ventrolateral PFC & -8 & 28 & 14 & 5.91 & 64 & FWE & 0.0380 \\
\hline
\end{tabular}

Total HF HRV differentiated neural responses to shocks (synchronous- delayed) within left insula ROI (voxel-level FDR). Increased HF HRV after delayed shocks (relative to synchronous shocks) was associated with increased neural response to delayed shocks (relative to synchronous) within right amygdala and periaqueductal gray. In addition to regions of interest, significant positive associations (FWE) within anterior cingulate cortex and left ventrolateral prefrontal cortex were observed.

reflect an individual's autonomic or affective style; participants with blood phobia and vasovagal syncope display exaggerated MSNA inhibition (Donadio et al., 2007). In our study increased HF HRV after delayed shocks was closely associated with increased functional activity within the PAG and amygdala. The PAG contributes to neural circuitry regulating anxiety, defensive motor and autonomic responses to surprising or threatening stimuli, including the initiation of nociceptive and antinociceptive reactions (Fields and Basbaum, 1999; Mason, 2005; Mobbs et al., 2007). Anatomically, PAG is connected reciprocally to insula, amygdala and medullary centers, including RVLM, in which cardiovascular responses are regulated (Behbehani, 1995). Caudal ventrolateral PAG columns generate depressor responses (Lovick, 1992), primarily altering blood pressure rather than heart rate (van der Plas et al., 1995). Likewise, neurons in the central nucleus of the amygdala, projecting monosynaptically to the RVLM cardiovascular nucleus, are activated by blood pressure decreases (Saha et al., 2005). Our observation that cardiac timing differences in HF HRV power after brief sensory stimuli are reflected in differences in functional activity within PAG and amygdala further strongly implicates these regions in autonomic integration of baroreceptor influences with stimulus processing. Subjects with greater parasympathetic HRV responses to delayed shocks also showed greater functional activity within the PAG and amygdala.

Our human neuroimaging data detail the central influence of afferent information from baroreceptors and may be interpreted in the light of existing literature from animal studies. Animal studies show afferent neural activity from baroreceptors, beginning at the upstroke of each pressure wave, is relayed via the NTS to the caudal ventrolateral medulla (CVLM). From there, inhibitory projections dampen descending sympatho-excitatory efferent outputs generated within the RVLM (Sved et al., 2001). Neurons within CVLM exhibit tight cardiac synchrony and reflect arterial blood pressure at each cardiac cycle (Schreihofer and Guyenet, 2003). CVLM neurons also project directly and indirectly (via hypothalamus) to the PAG. Via the direct projection, baroreceptor information arrives at the PAG $\sim 250-350 \mathrm{~ms}$ after ECG R-wave (Edwards et al., 2002). Additionally information regarding cutaneous stimulation may reach the PAG via inputs from the parabrachial nucleus. The PAG is known to play a crucial role in quiescence and nociception, attenuating reflexive responses to painful stimuli (Fields and Basbaum, 1999; Jänig, 2006), and may act as a bidirectional gateway inhibiting the ascending transmission of pain to cortical regions such as insula cortex (Millan, 1999). The present findings, in the light of these previous observations suggest the PAG to be a plausible neural substrate for the temporal integration of baroreceptor and nociceptive information in the control of blood pressure.
Our observations also find support from Lane et al. (2009), who demonstrated correlations between HF HRV and regional brain activity (also within the PAG and left insula) during emotional processing. Others have also reported associations between left insula activity and HF HRV (Lane et al., 2001; Critchley et al., 2003; Gianaros et al., 2004; Napadow et al., 2008). Reduced HF HRV may represent an endophenotypic marker with relevance to both anxiety and depressive disorders and to cardiovascular disease (Bleil et al., 2008; Thayer and Lane, 2009). Our findings suggest that the influence of cardiac timing (presumably reflecting alterations in cardiac afferent feedback) on differential neural responses within insula, prefrontal and brainstem centers are closely matched by an individual's parasympathetic vagal reactivity. Although it is tempting to speculate about the efferent versus afferent nature of these differences in functional brain activity, the origin of fMRI inferences in prolonged hemodynamic correlates of neuronal firing suggest that the observed effects represent the summed neural responses of initial stimulus processing and equilibrative representation and regulation of subsequent autonomic responses. In this case we would expect fMRI signatures to reflect both the initial efferent initiation, afferent representation and the evolving homeostatic regulation of cardiovascular responses. This, along with constitutional differences across subjects, may account for the absence of a robust association between peripheral blood pressure responses and neural or HRV measures. Together our findings highlight the distinct cortical representations of synchronous and delayed stimuli through functional coupling with amygdala and brainstem nuclei with implications for the subjective appraisal of external stimuli. Further, they suggest that individual differences in parasympathetic tone and reactivity may predict the influence of cardiac afferent traffic on the attenuation of central electrophysiological responses and blunted perception of nociceptive stimuli at different phases of the cardiac cycle (Dworkin et al., 1994; Rau et al., 1994; Edwards et al., 2001, 2002, 2008; Rau and Elbert, 2001; al'Absi et al., 2005; McIntyre et al., 2006, 2008).

In summary, our findings show that sensory processing depends on when stimuli are experienced in relation to heart beat timing; here particularly manifest as significant differences in evoked cardiovascular (blood pressure) arousal. We implicate brainstem, limbic and cortical brain regions in the integration of somatosensory and baroreceptor information and a role of individual sympathovagal balance in determining the influence of cardiac baroreceptor influences on central stimulus related processing. Our findings have direct relevance to understanding instrumental conditioning of essential hypertension (Rau and Elbert, 2001), pain perception (Dworkin et al., 1994) and individual differences in psychophysiological response tendencies (including, blood phobia) (Donadio et al., 2007). 


\section{References}

al'Absi M, France CR, Ring C, France J, Harju A, McIntyre D, Wittmers LE (2005) Nociception and baroreceptor stimulation in hypertensionprone men and women. Psychophysiology 42:83-91.

Angrilli A, Mini A, Mucha RF, Rau H (1997) The influence of low blood pressure and baroreceptor activity on pain responses. Physiol Behav 62:391-397.

Ashburner J, Friston KJ (2005) Unified segmentation. Neuroimage 26:839-851.

Behbehani MM (1995) Functional characteristics of the midbrain periaqueductal gray. Prog Neurobiol 46:575-605.

Berntson GG, Cacioppo JT, Quigley KS (1993) Respiratory sinus arrhythmia: autonomic origins, physiological mechanisms, and psychophysiological implications. Psychophysiology, 30:183-196.

Bleil ME, Gianaros PJ, Jennings JR, Flory JD, Manuck SB (2008) Trait negative affect: toward an integrated model of understanding psychological risk for impairment in cardiac autonomic function. Psychosom Med 70:328-337.

Brody S, Rau H (1994) Behavioral and psychophysiological predictors of self-monitored 19 month blood pressure change in normotensives. J Psychosom Res 38:885-891.

Caraffa-Braga E, Granata L, Pinotti O (1973) Changes in blood-flow distribution during acute emotional stress in dogs. Pflugers Arch 339:203-216.

Craig AD (2002) How do you feel? Interoception: the sense of the physiological condition of the body. Nat Rev Neurosci 3:655-666.

Critchley HD (2005) Neural mechanisms of autonomic, affective, and cognitive integration. J Comp Neurol 493:154-166.

Critchley HD, Mathias CJ, Josephs O, O'Doherty J, Zanini S, Dewar BK, Cipolotti L, Shallice T, Dolan RJ (2003) Human cingulate cortex and autonomic control: converging neuroimaging and clinical evidence. Brain 126:2139-2152.

Critchley HD, Wiens S, Rotshtein P, Ohman A, Dolan RJ (2004) Neural systems supporting interoceptive awareness. Nat Neurosci 7:189-195.

Deichmann R, Gottfried JA, Hutton C, Turner R (2003) Optimized EPI for fMRI studies of the orbitofrontal cortex. Neuroimage 19:430-441.

Donadio V, Kallio M, Karlsson T, Nordin M, Wallin BG (2002a) Inhibition of human muscle sympathetic activity by sensory stimulation. J Physiol 544:285-292.

Donadio V, Karlsson T, Elam M, Wallin BG (2002b) Interindividual differences in sympathetic and effector responses to arousal in humans. J Physiol 544:293-302.

Donadio V, Liguori R, Elam M, Karlsson T, Montagna P, Cortelli P, Baruzzi A, Wallin BG (2007) Arousal elicits exaggerated inhibition of sympathetic nerve activity in phobic syncope patients. Brain 130:1653-1662.

Droste C, Kardos A, Brody S, Greenlee MW, Roskamm H, Rau H (1994) Baroreceptor stimulation: pain perception and sensory thresholds. Biol Psychol 37:101-113.

Dworkin BR, Elbert T, Rau H, Birbaumer N, Pauli P, Droste C, Brunia CH (1994) Central effects of baroreceptor activation in humans: attenuation of skeletal reflexes and pain perception. Proc Natl Acad Sci U S A 91:6329-6333.

Eder DN, Elam M, Wallin BG (2009) Sympathetic nerve and cardiovascular responses to auditory startle and prepulse inhibition. Int J Psychophysiol 71:149-155.

Edwards L, Ring C, McIntyre D, Carroll D (2001) Modulation of the human nociceptive flexion reflex across the cardiac cycle. Psychophysiology 38:712-718.

Edwards L, McIntyre D, Carroll D, Ring C, Martin U (2002) The human nociceptive flexion reflex threshold is higher during systole than diastole. Psychophysiology 39:678-681.

Edwards L, McIntyre D, Carroll D, Ring C, France CR, Martin U (2003) Effects of artificial and natural baroreceptor stimulation on nociceptive responding and pain. Psychophysiology 40:762-769.

Edwards L, Inui K, Ring C, Wang X, Kakigi R (2008) Pain-related evoked potentials are modulated across the cardiac cycle. Pain 137:488-494.

Elbert T, Rockstroh B, Lutzenberger W, Kessler M, Pietrowsky R (1988) Baroreceptor stimulation alters pain sensation depending on tonic blood pressure. Psychophysiology 25:25-29.

Fields HL, Basbaum AI (1999) Central nervous system mechanisms of pain modulation. In: Textbook of pain (Wall PD, Melzack R, eds), pp 309-329. Edinburgh: Churchill Livingstone.
Forsyth RP (1972) Sympathetic nervous system control of distribution of cardiac output in unanesthetized monkeys. Fed Proc 31:1240-1244.

Friston KJ, Worsley KJ, Frackowiak RSJ, Mazziotta JC, Evans AC (1994) Assessing the significance of focal activations using their spatial extent. Hum Brain Mapp 1:214-220.

Gianaros P, Van Der Veen F, Jennings J (2004) Regional cerebral blood flow correlates with heart period and high-frequency heart period variability during working-memory tasks: implications for the cortical and subcortical regulation of cardiac autonomic activity. Psychophysiology 41:521-530.

Gianaros PJ, Jennings JR, Sheu LK, Derbyshire SW, Matthews KA (2007) Heightened functional neural activation to psychological stress covaries with exaggerated blood pressure reactivity. Hypertension 49:134-140.

Gianaros PJ, Sheu LK, Matthews KA, Jennings JR, Manuck SB, Hariri AR (2008) Individual differences in stressor-evoked blood pressure reactivity vary with activation, volume, and functional connectivity of the amygdala. J Neurosci 28:990-999.

Gilbey MP (2007) Sympathetic rhythms and nervous integration. Clin Exp Pharmacol Physiol 34:356-361.

Gilbey MP, Spyer KM (1993) Essential organization of the sympathetic nervous system. Baillieres Clin Endocrinol Metab 7:259-278.

Gray MA, Critchley HD (2007) Interoceptive basis to craving. Neuron 54:183-186.

Gray MA, Harrison NA, Wiens S, Critchley HD (2007) Modulation of emotional appraisal by false physiological feedback during fMRI. PLoS ONE 2:e546.

Hutton C, Bork A, Josephs O, Deichmann R, Ashburner J, Turner R (2002) Image distortion correction in fMRI: a quantitative evaluation. Neuroimage 16:217-240.

Jänig W (1985) Organization of the lumbar sympathetic outflow to skeletal muscle and skin of the cat hindlimb and tail. Rev Physiol Biochem Pharmacol 102:119-213.

Jänig W (2006) The integrative action of the autonomic nervous system: neurobiology of homeostasis. Cambridge, UK: Cambridge UP.

Kardos A, Rau H, Greenlee MW, Droste C, Brody S, Roskamm H (1994) Reduced pain during baroreceptor stimulation in patients with symptomatic and silent myocardial ischaemia. Cardiovasc Res 28:515-518.

Katsarava Z, Ayzenberg I, Sack F, Limmroth V, Diener HC, Kaube H (2006) A novel method of eliciting pain-related potentials by transcutaneous electrical stimulation. Headache 46:1511-1517.

Kober H, Barrett LF, Joseph J, Bliss-Moreau E, Lindquist K, Wager TD (2008) Functional grouping and cortical-subcortical interactions in emotion: a meta-analysis of neuroimaging studies. Neuroimage 42:998-1031.

Lane RD, Reiman EM, Ahern GL, Thayer JF (2001) Activity in medial prefrontal cortex correlates with vagal component of heart rate variability during emotion. Brain Cogn 47:97-100.

Lane RD, McRae K, Reiman EM, Chen K, Ahern GL, Thayer JF (2009) Neural correlates of heart rate variability during emotion. Neuroimage 44:213-222.

Lovick TA (1992) Inhibitory modulation of the cardiovascular defence response by the ventrolateral periaqueductal grey matter in rats. Exp Brain Res 89:133-139.

Mancia G, Mark AL (1983) The cardiovascular system. In: Handbook of physiology (Shepherd JT, Abboud FM, eds), pp 755-793. Bethesda, American Physiological Society.

Mason P (2005) Ventromedial medulla: pain modulation and beyond. J Comp Neurol 493:2-8.

McAllen RM, Malpas SC (1997) Sympathetic burst activity: characteristics and significance. Clin Exp Pharmacol Physiol 24:791-799.

McIntyre D, Edwards L, Ring C, Parvin B, Carroll D (2006) Systolic inhibition of nociceptive responding is moderated by arousal. Psychophysiology 43:314-319.

McIntyre D, Kavussanu M, Ring C (2008) Effects of arterial and cardiopulmonary baroreceptor activation on the upper limb nociceptive flexion reflex and electrocutaneous pain in humans. Pain 137:550-555.

Millan MJ (1999) The induction of pain: an integrative review. Prog Neurobiol 57:1-164.

Mini A, Rau H, Montoya P, Palomba D, Birbaumer N (1995) Baroreceptor cortical effects, emotions and pain. Int J Psychophysiol 19:67-77.

Mobbs D, Petrovic P, Marchant JL, Hassabis D, Weiskopf N, Seymour B, 
Dolan RJ, Frith CD (2007) When fear is near: threat imminence elicits prefrontal-periaqueductal gray shifts in humans. Science 317:1079-1083.

Napadow V, Dhond R, Conti G, Makris N, Brown EN, Barbieri R (2008) Brain correlates of autonomic modulation: combining heart rate variability with fMRI. Neuroimage 42:169-177.

Oppenheimer S (2007) Cortical control of the heart. Cleveland Clin J Med 74 (Suppl 1):S27-S29.

Rau H, Elbert T (2001) Psychophysiology of arterial baroreceptors and the etiology of hypertension. Biol Psychol 57:179-201.

Rau H, Brody S, Larbig W, Pauli P, Vöhringer M, Harsch B, Kröling P, Birbaumer N (1994) Effects of PRES baroreceptor stimulation on thermal and mechanical pain threshold in borderline hypertensives and normotensives. Psychophysiology 31:480-485.

Saha S, Drinkhill MJ, Moore JP, Batten TF (2005) Central nucleus of amygdala projections to rostral ventrolateral medulla neurones activated by decreased blood pressure. Eur J Neurosci 21:1921-1930.

Schreihofer AM, Guyenet PG (2003) Baro-activated neurons with pulsemodulated activity in the rat caudal ventrolateral medulla express GAD67 mRNA. J Neurophysiol 89:1265-1277.

Spyer KM (1999) Central nervous control of the cardiovascular system. In: Autonomic failure: a textbook of clinical disorders of the autonomic nervous system (Mathias CJ, Bannister R, eds), pp 45-55. Oxford: Oxford UP.
Sved AF, Ito S, Madden CJ, Stocker SD, Yajima Y (2001) Excitatory inputs to the RVLM in the context of the baroreceptor reflex. Ann N Y Acad Sci 940:247-258.

Task Force of the European Society of Cardiology and the North American Society of Pacing and Electrophysiology (1996) Heart rate variability: standards of measurement, physiological interpretation and clinical use. Circulation 93:1043-1065.

Thayer JF, Lane RD (2009) Claude Bernard and the heart-brain connection: further elaboration of a model of neurovisceral integration. Neurosci Biobehav Rev 33:81-88.

van der Plas J, Wiersinga-Post JE, Maes FW, Bohus B (1995) Cardiovascular effects and changes in midbrain periaqueductal gray neuronal activity induced by electrical stimulation of the hypothalamus in the rat. Brain Res Bull 37:645-656.

Wallin BG (2007) Interindividual differences in muscle sympathetic nerve activity: a key to new insight into cardiovascular regulation? Acta Physiol (Oxf) 190:265-275.

Wittling W, Block A, Genzel S, Schweiger E (1998) Hemisphere asymmetry in parasympathetic control of the heart. Neuropsychologia 36:461-468.

$\mathrm{Yu}$ YH, Blessing WW (1997) Cutaneous vasoconstriction in conscious rabbits during alerting responses detected by hippocampal theta-rhythm. Am J Physiol 272:R208-R216. 